\title{
The Homoleptic Carbonyls of the Second Row Transition Metals: Evaluation of Hartree-Fock and Density Functional Theory Methods
}

Xuejun Feng, ${ }^{\text {a* }}$ Jiande Gu, ${ }^{\mathrm{b}}$ Yaoming Xie, ${ }^{\mathrm{c}}$ R. Bruce King, ${ }^{\mathrm{c}}$ and Henry F. Schaefer III ${ }^{\mathrm{c} *}$

${ }^{a}$ School of Chemical and Material Engineering, Southern Yangtze University, Wuxi

214122, P. R. China,

${ }^{b}$ Drug Design \& Discovery Center, Shanghai Institute of Materia Medica, CAS

Shanghai 201203 P. R. China

${ }^{c}$ Center for Computational Chemistry and Department of Chemistry, University of

Georgia, Athens, Georgia 30602

\section{Supporting Information}

Table S1. The harmonic vibrational frequencies $\left(\right.$ in $^{-1}$ ) and their IR intensities (in $\mathrm{km} / \mathrm{mol})$ for $\mathrm{Mo}(\mathrm{CO})_{6}$.

Table S2. The harmonic vibrational frequencies $\left(\mathrm{in}^{-1}\right.$ ) and their IR intensities (in $\mathrm{km} / \mathrm{mol}$ ) for $\mathrm{Tc}_{2}(\mathrm{CO})_{10}$.

Table S3. The harmonic vibrational frequencies $\left(\mathrm{in}^{-1}\right.$ ) and their IR intensities (in $\mathrm{km} / \mathrm{mol})$ for $\mathrm{Ru}(\mathrm{CO})_{5}$.

Table S4. The harmonic vibrational frequencies $\left(\mathrm{in}^{-1}\right.$ ) and their IR intensities (in $\mathrm{km} / \mathrm{mol})$ for $\mathrm{Ru}_{3}(\mathrm{CO})_{12}\left(D_{3 h}\right.$ unbridged isomer)

Table S5. The harmonic vibrational frequencies $\left(\mathrm{in}^{-1}\right.$ ) and their IR intensities (in $\mathrm{km} / \mathrm{mol})$ for $\mathrm{Ru}_{3}(\mathrm{CO})_{12}\left(C_{2 v}\right.$ dibridged isomer $)$.

Table S6. The harmonic vibrational frequencies $\left(\right.$ in $^{-1}$ ) and their IR intensities (in $\mathrm{km} / \mathrm{mol})$ for $\mathrm{Rh}_{4}(\mathrm{CO})_{12}\left(C_{3 v}\right.$ tribridged isomer).

Table S7. The harmonic vibrational frequencies $\left(\mathrm{in}^{-1}\right.$ ) and their IR intensities (in $\mathrm{km} / \mathrm{mol}$ ) for $\mathrm{Rh}_{4}(\mathrm{CO})_{12}\left(T_{d}\right.$ unbridged isomer).

Table S8. The harmonic vibrational frequencies $\left(\mathrm{in}^{-1}\right.$ ) and their IR intensities (in $\mathrm{km} / \mathrm{mol}$ ) for $\mathrm{Rh}_{6}(\mathrm{CO})_{16}\left(T_{d}\right.$ tetrabridged isomer). 
Table S1. The harmonic vibrational frequencies (in $\mathrm{cm}^{-1}$ ) and their IR intensities (in $\mathrm{km} / \mathrm{mol}$ ) for $\mathrm{Mo}(\mathrm{CO})_{6}$.

\begin{tabular}{ccccccccc}
\hline & \multicolumn{2}{c}{ B3LYP } & \multicolumn{2}{c}{ BP86 } & \multicolumn{2}{c}{ HF } & \multicolumn{2}{c}{ MPW1PW91 } \\
\hline $\mathrm{a}_{1 \mathrm{~g}}$ & 407 & $(0)$ & 416 & $(0)$ & 362 & $(0)$ & 423 & $(0)$ \\
& 2175 & $(0)$ & 2092 & $(0)$ & 2415 & $(0)$ & 2220 & $(0)$ \\
$\mathrm{e}_{\mathrm{g}}$ & 393 & $(0)$ & 403 & $(0)$ & 353 & $(0)$ & 411 & $(0)$ \\
& 2080 & $(0)$ & 2001 & $(0)$ & 2326 & $(0)$ & 2125 & $(0)$ \\
$\mathrm{t}_{1 \mathrm{~g}}$ & 341 & $(0)$ & 334 & $(0)$ & 353 & $(0)$ & 351 & $(0)$ \\
$\mathrm{t}_{1 \mathrm{u}}$ & 88 & $(1)$ & 84 & $(1)$ & 100 & $(2)$ & 90 & $(1)$ \\
& 381 & $(51)$ & 394 & $(34)$ & 342 & $(97)$ & 401 & $(50)$ \\
& 611 & $(112)$ & 602 & $(96)$ & 627 & $(181)$ & 629 & $(127)$ \\
& 2057 & $(2085)$ & 1979 & $(1786)$ & 2298 & $(2718)$ & 2099 & $(2139)$ \\
$\mathrm{t}_{2 \mathrm{~g}}$ & 81 & $(0)$ & 78 & $(0)$ & 88 & $(0)$ & 82 & $(0)$ \\
& 484 & $(0)$ & 469 & $(0)$ & 516 & $(0)$ & 496 & $(0)$ \\
$\mathrm{t}_{2 \mathrm{u}}$ & 57 & $(0)$ & 53 & $(0)$ & 66 & $(0)$ & 57 & $(0)$ \\
& 519 & $(0)$ & 508 & $(0)$ & 534 & $(0)$ & 530 & $(0)$ \\
\hline
\end{tabular}


Table S2. The harmonic vibrational frequencies (in $\mathrm{cm}^{-1}$ ) and their IR intensities (in $\mathrm{km} / \mathrm{mol}$ ) for $\mathrm{Tc}_{2}(\mathrm{CO})_{10}$.

\begin{tabular}{|c|c|c|c|c|c|c|c|c|}
\hline \multirow{2}{*}{$\mathrm{a}_{1}$} & \multicolumn{2}{|c|}{ B3LYP } & \multicolumn{2}{|c|}{ BP86 } & \multicolumn{2}{|c|}{$\mathrm{HF}$} & \multicolumn{2}{|c|}{ MPW1PW91 } \\
\hline & 87 & (0) & 84 & (0) & 92 & (0) & 91 & (0) \\
\hline & 138 & (0) & 136 & $(0)$ & 147 & $(0)$ & 149 & (0) \\
\hline & 434 & (0) & 442 & (0) & 386 & (0) & 451 & (0) \\
\hline & 464 & $(0)$ & 473 & $(0)$ & 425 & $(0)$ & 486 & (0) \\
\hline & 640 & (0) & 629 & (0) & 663 & (0) & 659 & (0) \\
\hline & 2065 & (0) & 1989 & (0) & 2306 & (0) & 2108 & (0) \\
\hline & 2182 & $(0)$ & 2099 & (0) & 2423 & (0) & 2228 & (0) \\
\hline$a_{2}$ & 354 & (0) & 348 & (0) & 365 & (0) & 365 & (0) \\
\hline$b_{1}$ & 33 & (0) & 33 & (0) & 39 & (0) & 37 & (0) \\
\hline & 357 & (0) & 350 & $(0)$ & 369 & (0) & 368 & $(0)$ \\
\hline $\mathrm{b}_{2}$ & 102 & (3) & 98 & (3) & 110 & (6) & 104 & (3) \\
\hline & 430 & (5) & 439 & (4) & 366 & (54) & 446 & (6) \\
\hline & 463 & (4) & 472 & $(0)$ & 421 & (70) & 484 & (3) \\
\hline & 618 & $(579)$ & 612 & (494) & 627 & (833) & 636 & $(601)$ \\
\hline & 2052 & (1163) & 1978 & (1098) & 2292 & (601) & 2096 & (1280) \\
\hline & 2120 & (1377) & 2047 & (1057) & 2329 & (3306) & 2166 & (1295) \\
\hline$e_{1}$ & 51 & $(0)$ & 48 & $(0)$ & 55 & (0) & 52 & $(0)$ \\
\hline & 86 & (0) & 83 & (0) & 96 & (0) & 89 & $(0)$ \\
\hline & 97 & (1) & 94 & $(0)$ & 108 & (1) & 99 & (0) \\
\hline & 402 & (27) & 398 & (0) & 372 & (81) & 419 & (17) \\
\hline & 416 & (32) & 421 & (41) & 442 & (12) & 433 & (40) \\
\hline & 536 & (11) & 521 & (9) & 574 & (7) & 553 & (8) \\
\hline & 610 & (150) & 599 & (129) & 630 & $(231)$ & 627 & (167) \\
\hline & 2082 & (2712) & 2002 & (2336) & 2329 & (3539) & 2125 & (2768) \\
\hline$e_{2}$ & 67 & $(0)$ & 62 & $(0)$ & 77 & (0) & 67 & (0) \\
\hline & 90 & (0) & 87 & (0) & 100 & (0) & 92 & $(0)$ \\
\hline & 426 & (0) & 433 & (0) & 379 & (0) & 443 & (0) \\
\hline & 482 & (0) & 468 & (0) & 528 & (0) & 497 & (0) \\
\hline & 507 & (0) & 490 & (0) & 541 & (0) & 518 & $(0)$ \\
\hline & 2087 & (0) & 2010 & (0) & 2325 & (0) & 2132 & (0) \\
\hline$e_{3}$ & 51 & (0) & 46 & (0) & 62 & (0) & 52 & (0) \\
\hline & 80 & (0) & 76 & (0) & 88 & (0) & 81 & (0) \\
\hline & 99 & (0) & 95 & (0) & 111 & (0) & 101 & (0) \\
\hline & 395 & (0) & 387 & (0) & 365 & (0) & 410 & (0) \\
\hline & 405 & (0) & 416 & (0) & 429 & (0) & 424 & (0) \\
\hline & 534 & (0) & 520 & (0) & 567 & (0) & 549 & (0) \\
\hline & 608 & (0) & 597 & (0) & 629 & (0) & 625 & (0) \\
\hline & 2051 & (0) & 1972 & (0) & 2300 & (0) & 2093 & (0) \\
\hline
\end{tabular}


Table S3. The harmonic vibrational frequencies $\left(\mathrm{in}^{-1}\right.$ ) and their IR intensities (in $\mathrm{km} / \mathrm{mol})$ for $\mathrm{Ru}(\mathrm{CO})_{5}$.

\begin{tabular}{ccccccccc}
\hline & \multicolumn{2}{c}{ B3LYP } & \multicolumn{2}{c}{ BP86 } & \multicolumn{3}{c}{ HF } & \multicolumn{2}{c}{ MPWPW91 } \\
\hline $\mathrm{a}_{1}{ }^{\prime}$ & 420 & $(0)$ & 431 & $(0)$ & 365 & $(0)$ & 441 & $(0)$ \\
& 450 & $(0)$ & 461 & $(0)$ & 378 & $(0)$ & 468 & $(0)$ \\
& 2090 & $(0)$ & 2009 & $(0)$ & 2342 & $(0)$ & 2135 & $(0)$ \\
& 2179 & $(0)$ & 2096 & $(0)$ & 2428 & $(0)$ & 2225 & $(0)$ \\
$\mathrm{a}_{2}{ }^{\prime}$ & 333 & $(0)$ & 323 & $(0)$ & 368 & $(0)$ & 349 & $(0)$ \\
& 91 & $(0)$ & 87 & $(0)$ & 101 & $(1)$ & 94 & $(0)$ \\
& 411 & $(24)$ & 427 & $(12)$ & 352 & $(47)$ & 432 & $(22)$ \\
& 532 & $(114)$ & 527 & $(113)$ & 545 & $(96)$ & 552 & $(126)$ \\
& 2100 & $(1474)$ & 2017 & $(1289)$ & 2372 & $(1715)$ & 2144 & $(1513)$ \\
$\mathrm{e}^{\prime}$ & 46 & $(0)$ & 45 & $(0)$ & 53 & $(1)$ & 48 & $(0)$ \\
& 93 & $(0)$ & 89 & $(0)$ & 102 & $(0)$ & 94 & $(0)$ \\
& 380 & $(43)$ & 387 & $(28)$ & 348 & $(72)$ & 402 & $(41)$ \\
& 451 & $(0)$ & 449 & $(0)$ & 467 & $(9)$ & 469 & $(0)$ \\
& 611 & $(119)$ & 602 & $(111)$ & 611 & $(136)$ & 627 & $(133)$ \\
& 2063 & $(1314)$ & 1985 & $(1143)$ & 2301 & $(1793)$ & 2106 & $(1360)$ \\
$\mathrm{e}^{\prime \prime}$ & 86 & $(0)$ & 82 & $(0)$ & 93 & $(0)$ & 87 & $(0)$ \\
& 324 & $(0)$ & 315 & $(0)$ & 338 & $(0)$ & 338 & $(0)$ \\
& 505 & $(0)$ & 489 & $(0)$ & 535 & $(0)$ & 518 & $(0)$ \\
\hline
\end{tabular}


Table S4. The harmonic vibrational frequencies $\left(\mathrm{in}^{-1}\right.$ ) and their IR intensities (in $\mathrm{km} / \mathrm{mol}$ ) for $\mathrm{Ru}_{3}(\mathrm{CO})_{12}\left(\mathrm{D}_{3 h}\right.$ unbridged isomer)

\begin{tabular}{|c|c|c|c|c|c|c|c|c|}
\hline \multirow{2}{*}{$\mathrm{a}_{1}{ }^{\prime}$} & \multicolumn{2}{|c|}{ B3LYP } & \multicolumn{2}{|c|}{ BP86 } & \multicolumn{2}{|c|}{$\mathrm{HF}$} & \multicolumn{2}{|c|}{ MPW1PW91 } \\
\hline & 75 & (0) & 75 & (0) & 84 & (0) & 80 & (0) \\
\hline & 149 & (0) & 144 & (0) & 162 & (0) & 155 & (0) \\
\hline & 170 & (0) & 169 & (0) & 181 & (0) & 185 & (0) \\
\hline & 439 & (0) & 444 & (0) & 396 & (0) & 456 & (0) \\
\hline & 467 & (0) & 479 & (0) & 417 & (0) & 490 & (0) \\
\hline & 504 & (0) & 493 & (0) & 541 & (0) & 522 & (0) \\
\hline & 609 & (0) & 593 & (0) & 657 & (0) & 630 & (0) \\
\hline & 2098 & (0) & 2017 & (0) & 2345 & (0) & 2142 & (0) \\
\hline & 2179 & (0) & 2094 & (0) & 2429 & (0) & 2225 & (0) \\
\hline & -15 & (0) & -18 & (0) & -14 & (0) & -18 & (0) \\
\hline & 87 & (0) & 83 & (0) & 95 & (0) & 88 & (0) \\
\hline & 388 & (0) & 380 & (0) & 412 & (0) & 403 & (0) \\
\hline & 521 & (0) & 506 & (0) & 558 & (0) & 537 & (0) \\
\hline \multirow[t]{12}{*}{$\mathrm{a}_{2}{ }^{\prime}$} & 29 & (0) & 23 & (0) & 46 & (0) & 27 & (0) \\
\hline & 94 & (0) & 91 & (0) & 92 & $(0)$ & 94 & (0) \\
\hline & 393 & (0) & 383 & (0) & 374 & (0) & 410 & (0) \\
\hline & 426 & (0) & 443 & (0) & 440 & (0) & 451 & (0) \\
\hline & 567 & (0) & 561 & (0) & 566 & $(0)$ & 585 & (0) \\
\hline & 2056 & (0) & 1979 & (0) & 2302 & $(0)$ & 2100 & (0) \\
\hline & 86 & (0) & 83 & (0) & 96 & (1) & 88 & (0) \\
\hline & 147 & (0) & 143 & (0) & 160 & (0) & 154 & (0) \\
\hline & 404 & (13) & 403 & (1) & 379 & (79) & 421 & (9) \\
\hline & 450 & (98) & 451 & (86) & 471 & (110) & 473 & (106) \\
\hline & 557 & (83) & 541 & (74) & 606 & (99) & 576 & (89) \\
\hline & 2098 & (2989) & 2015 & (2526) & 2351 & (4225) & 2140 & (3052) \\
\hline \multirow[t]{15}{*}{$\mathrm{e}^{\prime}$} & 39 & (0) & 36 & (0) & 51 & (0) & 41 & (0) \\
\hline & 70 & (0) & 68 & (0) & 76 & (1) & 72 & (0) \\
\hline & 101 & (1) & 97 & (1) & 109 & (1) & 103 & (1) \\
\hline & 110 & (0) & 107 & (0) & 120 & (2) & 117 & (0) \\
\hline & 140 & (1) & 138 & (1) & 151 & (5) & 150 & (1) \\
\hline & 381 & (2) & 370 & (1) & 376 & (40) & 400 & (2) \\
\hline & 428 & (0) & 428 & (0) & 385 & (2) & 444 & (0) \\
\hline & 435 & (2) & 447 & (11) & 408 & (90) & 459 & (4) \\
\hline & 457 & (10) & 462 & (14) & 435 & (1) & 478 & (14) \\
\hline & 473 & (49) & 484 & (17) & 489 & (144) & 497 & (45) \\
\hline & 586 & (72) & 572 & (54) & 618 & (190) & 604 & (86) \\
\hline & 609 & $(354)$ & 599 & (323) & 642 & $(356)$ & 629 & (364) \\
\hline & 2071 & (33) & 1992 & (19) & 2307 & (230) & 2115 & (10) \\
\hline & 2074 & $(460)$ & 1995 & (560) & 2320 & (34) & 2117 & (488) \\
\hline & 2118 & (2345) & 2042 & (1789) & 2348 & (4332) & 2163 & (2385) \\
\hline \multirow[t]{10}{*}{ e" } & 41 & (0) & 32 & (0) & 54 & (0) & 36 & (0) \\
\hline & 72 & (0) & 68 & (0) & 80 & (0) & 72 & (0) \\
\hline & 94 & (0) & 90 & (0) & 106 & (0) & 96 & (0) \\
\hline & 116 & (0) & 113 & (0) & 127 & (0) & 122 & (0) \\
\hline & 380 & (0) & 371 & (0) & 371 & (0) & 395 & (0) \\
\hline & 392 & (0) & 389 & (0) & 408 & (0) & 408 & (0) \\
\hline & 450 & (0) & 455 & (0) & 457 & (0) & 471 & (0) \\
\hline & 522 & (0) & 506 & (0) & 566 & (0) & 539 & (0) \\
\hline & 556 & (0) & 543 & (0) & 594 & (0) & 575 & (0) \\
\hline & 2055 & $(0)$ & 1974 & (0) & 2310 & $(0)$ & 2097 & (0) \\
\hline
\end{tabular}


Table S5. The harmonic vibrational frequencies (in $\mathrm{cm}-1$ ) and their IR intensities (in $\mathrm{km} / \mathrm{mol}$ ) for $\mathrm{Ru}_{3}(\mathrm{CO})_{12}\left(C_{2 v}\right.$ dibridged isomer).

\begin{tabular}{|c|c|c|c|c|c|c|c|c|}
\hline & \multicolumn{2}{|c|}{ B3LYP } & \multicolumn{2}{|c|}{ BP86 } & \multicolumn{2}{|c|}{$\mathrm{HF}$} & \multicolumn{2}{|c|}{ MPW1PW91 } \\
\hline \multirow[t]{24}{*}{$\mathrm{a}_{1}$} & 53 & (0) & 51 & (0) & 57 & (1) & 54 & (0) \\
\hline & 74 & (0) & 73 & (0) & 78 & (1) & 76 & (0) \\
\hline & 80 & (0) & 77 & (0) & 89 & (0) & 82 & (0) \\
\hline & 87 & (0) & 85 & (0) & 94 & (1) & 90 & (0) \\
\hline & 98 & (0) & 94 & (0) & 107 & (0) & 100 & (0) \\
\hline & 109 & (0) & 105 & (0) & 126 & (1) & 115 & (1) \\
\hline & 153 & (0) & 151 & (0) & 167 & (3) & 165 & (0) \\
\hline & 198 & (0) & 195 & (0) & 213 & (2) & 212 & (0) \\
\hline & 357 & (1) & 359 & (0) & 349 & (8) & 378 & (0) \\
\hline & 405 & (23) & 393 & (17) & 374 & (0) & 421 & (25) \\
\hline & 428 & (2) & 438 & (4) & 383 & (80) & 449 & (1) \\
\hline & 442 & (3) & 449 & (6) & 396 & (50) & 461 & (7) \\
\hline & 451 & (0) & 456 & (2) & 419 & (0) & 473 & (0) \\
\hline & 455 & (5) & 469 & (0) & 441 & (32) & 479 & (2) \\
\hline & 470 & (20) & 475 & (4) & 493 & (130) & 492 & (16) \\
\hline & 513 & (5) & 502 & (4) & 542 & (7) & 529 & (5) \\
\hline & 561 & (24) & 549 & (37) & 585 & (23) & 579 & (26) \\
\hline & 583 & (260) & 574 & (207) & 621 & (426) & 602 & (297) \\
\hline & 596 & (99) & 582 & (99) & 637 & (5) & 615 & (87) \\
\hline & 1945 & (242) & 1874 & (186) & 2117 & (637) & 1984 & (245) \\
\hline & 2078 & (598) & 1997 & (695) & 2320 & (627) & 2122 & (630) \\
\hline & 2092 & $(174)$ & 2010 & (131) & 2343 & (585) & 2136 & (135) \\
\hline & 2119 & (1898) & 2040 & (1385) & 2369 & (3111) & 2163 & (1958) \\
\hline & 2171 & (62) & 2085 & (69) & 2428 & (2) & 2217 & (58) \\
\hline \multirow[t]{14}{*}{$\mathrm{a}_{2}$} & 40 & (0) & 36 & (0) & $160 \mathrm{i}$ & & 13 & (0) \\
\hline & 52 & (0) & 41 & (0) & 51 & (0) & 40 & (0) \\
\hline & 81 & (0) & 51 & (0) & 58 & (0) & 54 & (0) \\
\hline & 94 & (0) & 80 & (0) & 86 & (0) & 83 & (0) \\
\hline & -39 & (0) & 93 & (0) & 102 & (0) & 98 & (0) \\
\hline & 158 & (0) & 163 & (0) & 159 & (0) & 173 & (0) \\
\hline & 297 & (0) & 292 & (0) & 297 & (0) & 309 & (0) \\
\hline & 380 & (0) & 370 & (0) & 395 & (0) & 396 & (0) \\
\hline & 401 & (0) & 392 & (0) & 407 & (0) & 417 & (0) \\
\hline & 434 & (0) & 441 & (0) & 441 & (0) & 455 & (0) \\
\hline & 488 & (0) & 476 & (0) & 523 & (0) & 504 & (0) \\
\hline & 516 & (0) & 501 & (0) & 557 & (0) & 533 & (0) \\
\hline & 587 & (0) & 577 & (0) & 618 & (0) & 608 & (0) \\
\hline & 2082 & (0) & 2001 & (0) & 2340 & (0) & 2127 & (0) \\
\hline \multirow[t]{14}{*}{$b_{1}$} & 42 & (0) & 38 & (0) & 49 & (2) & 42 & (0) \\
\hline & 68 & (0) & 65 & (0) & 77 & (0) & 70 & (0) \\
\hline & 72 & (0) & 67 & (0) & 81 & (1) & 71 & (0) \\
\hline & 85 & (0) & 82 & (0) & 87 & (4) & 87 & (0) \\
\hline & 90 & (0) & 87 & (0) & 102 & (0) & 93 & (0) \\
\hline & 107 & (0) & 103 & (0) & 123 & (0) & 112 & (0) \\
\hline & 353 & (8) & 355 & (3) & 319 & (132) & 375 & (6) \\
\hline & 382 & (0) & 375 & (0) & 371 & (22) & 399 & (0) \\
\hline & 395 & (7) & 390 & (1) & 384 & (46) & 411 & (5) \\
\hline & 427 & (24) & 420 & (1) & 424 & (6) & 445 & (3) \\
\hline & 431 & (0) & 438 & (6) & 445 & (0) & 451 & (10) \\
\hline & 442 & (57) & 445 & (53) & 468 & (79) & 462 & (66) \\
\hline & 523 & (0) & 506 & (0) & 555 & (125) & 538 & (2) \\
\hline & 537 & (139) & 527 & $(118)$ & 575 & (64) & 556 & (153) \\
\hline
\end{tabular}




\begin{tabular}{ccccccccc}
\hline & 548 & $(0)$ & 537 & $(9)$ & 591 & $(0)$ & 567 & $(0)$ \\
& 1897 & $(846)$ & 1844 & $(616)$ & 1903 & $(3093)$ & 1935 & $(880)$ \\
2069 & $(0)$ & 1987 & $(3)$ & 2330 & $(3)$ & 2112 & $(4)$ \\
& 2098 & $(2420)$ & 2015 & $(2069)$ & 2354 & $(3182)$ & 2142 & $(2453)$ \\
& 43 & $(0)$ & 41 & $(0)$ & 50 & $(0)$ & 44 & $(0)$ \\
77 & $(0)$ & 75 & $(0)$ & 62 & $(1)$ & 79 & $(0)$ \\
87 & $(0)$ & 85 & $(0)$ & 84 & $(0)$ & 90 & $(0)$ \\
93 & $(0)$ & 90 & $(0)$ & 97 & $(0)$ & 95 & $(0)$ \\
94 & $(0)$ & 93 & $(0)$ & 103 & $(0)$ & 98 & $(0)$ \\
& 144 & $(1)$ & 142 & $(1)$ & 153 & $(1)$ & 156 & $(1)$ \\
215 & $(7)$ & 213 & $(6)$ & 225 & $(3)$ & 225 & $(6)$ \\
388 & $(0)$ & 378 & $(2)$ & 344 & $(7)$ & 406 & $(0)$ \\
& $(15)$ & 397 & $(11)$ & 373 & $(12)$ & 415 & $(11)$ \\
& 424 & $(53)$ & 434 & $(58)$ & 384 & $(115)$ & 446 & $(61)$ \\
444 & $(21)$ & 453 & $(4)$ & 428 & $(16)$ & 468 & $(16)$ \\
449 & $(7)$ & 462 & $(17)$ & 438 & $(1)$ & 473 & $(7)$ \\
486 & $(12)$ & 486 & $(7)$ & 492 & $(0)$ & 505 & $(10)$ \\
547 & $(75)$ & 537 & $(40)$ & 568 & $(276)$ & 565 & $(88)$ \\
574 & $(173)$ & 563 & $(135)$ & 603 & $(16)$ & 594 & $(96)$ \\
& $(389)$ & 574 & $(408)$ & 633 & $(486)$ & 607 & $(508)$ \\
& 587 & $(72)$ & 1985 & $(80)$ & 2317 & $(15)$ & 2109 & $(78)$ \\
& 2065 & $(154)$ & 2001 & $(146)$ & 2352 & $(1316)$ & 2127 & $(167)$ \\
2083 & $(2454)$ & 2038 & $(2087)$ & 2370 & $(2567)$ & 2165 & $(2482)$ \\
\hline & & & & & & &
\end{tabular}


Table S6. The harmonic vibrational frequencies (in $\mathrm{cm}^{-1}$ ) and their IR intensities (in $\mathrm{km} / \mathrm{mol}$ ) for $\mathrm{Rh}_{4}(\mathrm{CO})_{12}\left(C_{3 v}\right.$ tribridged isomer).

\begin{tabular}{|c|c|c|c|c|c|c|c|c|}
\hline \multirow[b]{2}{*}{$a_{1}$} & \multicolumn{2}{|c|}{ B3LYP } & \multicolumn{2}{|c|}{ BP86 } & \multicolumn{2}{|c|}{$\mathrm{HF}$} & \multicolumn{2}{|c|}{ MPW1PW91 } \\
\hline & 40 & (0) & 38 & (0) & 45 & (0) & 41 & (0) \\
\hline & 74 & (0) & 72 & (0) & 78 & (0) & 76 & (0) \\
\hline & 81 & (0) & 78 & (0) & 87 & (0) & 84 & (0) \\
\hline & 87 & (0) & 84 & (0) & 97 & (0) & 91 & (0) \\
\hline & 166 & (2) & 164 & (1) & 173 & (3) & 179 & (2) \\
\hline & 211 & (0) & 210 & (0) & 214 & (10) & 227 & (0) \\
\hline & 354 & (10) & 349 & (8) & 316 & (25) & 372 & (10) \\
\hline & 366 & (0) & 366 & (0) & 336 & (80) & 388 & (0) \\
\hline & 405 & (8) & 428 & (15) & 349 & (2) & 432 & (8) \\
\hline & 418 & (30) & 441 & (16) & 361 & (1) & 444 & (27) \\
\hline & 444 & (9) & 460 & (13) & 389 & (1) & 467 & (12) \\
\hline & 487 & (9) & 480 & (11) & 500 & (87) & 507 & (1) \\
\hline & 499 & $(200)$ & 495 & (190) & 518 & (43) & 520 & (235) \\
\hline & 517 & (6) & 515 & (0) & 526 & (118) & 538 & (5) \\
\hline & 1986 & (9) & 1903 & (5) & 2211 & (88) & 2026 & (8) \\
\hline & 2095 & $(362)$ & 2010 & (514) & 2348 & (509) & 2139 & (348) \\
\hline & 2124 & (2512) & 2039 & (1935) & 2391 & (3111) & 2169 & $(2572)$ \\
\hline & 2160 & (0) & 2071 & (1) & 2431 & (168) & 2206 & (0) \\
\hline $\mathrm{a}_{2}$ & 39 & (0) & 36 & (0) & 46 & (0) & 39 & (0) \\
\hline & 57 & (0) & 56 & (0) & 60 & (0) & 58 & (0) \\
\hline & 83 & (0) & 81 & (0) & 87 & (0) & 85 & (0) \\
\hline & 193 & (0) & 203 & (0) & 185 & (0) & 208 & (0) \\
\hline & 329 & (0) & 323 & (0) & 357 & (0) & 347 & (0) \\
\hline & 356 & (0) & 353 & (0) & 365 & (0) & 372 & (0) \\
\hline & 413 & (0) & 408 & (0) & 420 & (4) & 431 & (0) \\
\hline & 565 & (0) & 564 & (0) & 561 & (0) & 584 & (0) \\
\hline $\mathrm{e}$ & 34 & (0) & 33 & (0) & $194 i$ & & 35 & (0) \\
\hline & 55 & (0) & 55 & (0) & 37 & (0) & 57 & (0) \\
\hline & 65 & (0) & 62 & (0) & 62 & (1) & 66 & (0) \\
\hline & 69 & (0) & 67 & (0) & 70 & (0) & 71 & (0) \\
\hline & 74 & (0) & 73 & (0) & 76 & (0) & 77 & (0) \\
\hline & 77 & (0) & 75 & (0) & 80 & (0) & 79 & (0) \\
\hline & 85 & (0) & 83 & (0) & 91 & (0) & 88 & (0) \\
\hline & 122 & (0) & 121 & (0) & 107 & (0) & 130 & (0) \\
\hline & 164 & (0) & 163 & (0) & 137 & (1) & 177 & (0) \\
\hline & 192 & (4) & 198 & (2) & 179 & (1) & 203 & (3) \\
\hline & 321 & (2) & 336 & (5) & 218 & (26) & 344 & (1) \\
\hline & 335 & (14) & 346 & (3) & 275 & (1) & 356 & (12) \\
\hline & 357 & (14) & 358 & (19) & 323 & (1) & 376 & (16) \\
\hline & 364 & (3) & 364 & (2) & 330 & (51) & 383 & (2) \\
\hline & 387 & (6) & 383 & (8) & 353 & (6) & 406 & (6) \\
\hline & 411 & (1) & 429 & (15) & 391 & (6) & 438 & (3) \\
\hline & 419 & (6) & 437 & (10) & 403 & (0) & 445 & (7) \\
\hline & 435 & (11) & 444 & (42) & 420 & (0) & 456 & (17) \\
\hline & 452 & (75) & 457 & (13) & 454 & $(170)$ & 474 & (75) \\
\hline & 487 & (1) & 480 & (4) & 489 & $(0)$ & 506 & (3) \\
\hline & 497 & (71) & 497 & (75) & 511 & (2) & 519 & (71) \\
\hline & 520 & (46) & 521 & (38) & 513 & (101) & 542 & (64) \\
\hline & 1953 & $(867)$ & 1879 & $(689)$ & 2157 & (1625) & 1990 & (902) \\
\hline & 2080 & (31) & 1994 & (51) & 2339 & $(0)$ & 2124 & (49) \\
\hline & 2096 & (313) & 2009 & (283) & 2372 & (1639) & 2140 & $(300)$ \\
\hline & 2123 & (1690) & 2038 & (1407) & 2392 & (1280) & 2169 & $(1720)$ \\
\hline
\end{tabular}


Table S7. The harmonic vibrational frequencies (in $\mathrm{cm}^{-1}$ ) and their IR intensities (in $\mathrm{km} / \mathrm{mol})$ for $\mathrm{Rh}_{4}(\mathrm{CO})_{12}\left(T_{d}\right.$ unbridged isomer).

\begin{tabular}{|c|c|c|c|c|c|c|c|c|}
\hline \multirow[b]{2}{*}{$\mathrm{e}$} & \multicolumn{2}{|c|}{ B3LYP } & \multicolumn{2}{|c|}{ BP86 } & \multicolumn{2}{|c|}{$\mathrm{HF}$} & \multicolumn{2}{|c|}{ MPW1PW91 } \\
\hline & 55 & $(0)$ & 53 & (0) & 62 & (0) & 57 & $(0)$ \\
\hline & 73 & (0) & 71 & (0) & 83 & (0) & 78 & (0) \\
\hline & 139 & (0) & 135 & (0) & 160 & $(0)$ & 148 & $(0)$ \\
\hline & 384 & (0) & 377 & $(0)$ & 354 & $(0)$ & 400 & (0) \\
\hline & 431 & $(0)$ & 448 & (0) & 435 & (0) & 458 & (0) \\
\hline & 470 & (0) & 465 & (0) & 488 & $(0)$ & 493 & (0) \\
\hline & 2076 & (0) & 1990 & $(0)$ & 2344 & $(0)$ & 2118 & $(0)$ \\
\hline \multirow[t]{5}{*}{$a_{1}$} & 94 & (0) & 91 & $(0)$ & 109 & $(0)$ & 100 & (0) \\
\hline & 233 & (0) & 229 & (0) & 252 & (0) & 250 & (0) \\
\hline & 423 & (0) & 442 & (0) & 356 & (0) & 452 & (0) \\
\hline & 512 & (0) & 509 & $(0)$ & 539 & $(0)$ & 533 & (0) \\
\hline & 2164 & (0) & 2075 & (0) & 2425 & $(0)$ & 2207 & (0) \\
\hline \multirow[t]{2}{*}{$\mathrm{a}_{2}$} & 27 & (0) & 22 & $(0)$ & 36 & $(0)$ & 28 & (0) \\
\hline & 354 & (0) & 347 & (0) & 383 & (0) & 372 & (0) \\
\hline \multirow[t]{8}{*}{$\mathrm{t}_{1}$} & 15 & (0) & 20 & (0) & -255 & (0) & -10 & (0) \\
\hline & 46 & (0) & 45 & (0) & 52 & (0) & 50 & (0) \\
\hline & 75 & (0) & 75 & (0) & 73 & $(0)$ & 79 & (0) \\
\hline & 304 & (0) & 317 & (0) & 151 & (0) & 328 & (0) \\
\hline & 338 & (0) & 332 & (0) & 364 & (0) & 356 & (0) \\
\hline & 390 & (0) & 406 & (0) & 383 & (0) & 413 & (0) \\
\hline & 458 & (0) & 461 & (0) & 446 & (0) & 480 & (0) \\
\hline & 2057 & (0) & 1974 & (0) & 2309 & (0) & 2099 & (0) \\
\hline \multirow[t]{11}{*}{$t_{2}$} & 72 & (0) & 72 & (0) & 85 & (1) & 79 & (1) \\
\hline & 81 & (0) & 79 & (0) & 92 & (0) & 87 & (0) \\
\hline & 87 & (0) & 85 & (0) & 100 & (1) & 92 & (1) \\
\hline & 180 & (2) & 176 & (1) & 198 & (9) & 192 & (2) \\
\hline & 396 & (32) & 390 & (27) & 328 & (76) & 415 & (34) \\
\hline & 416 & (6) & 432 & (0) & 364 & (45) & 443 & (2) \\
\hline & 436 & (10) & 454 & (7) & 439 & (21) & 462 & (10) \\
\hline & 474 & (81) & 466 & (81) & 500 & (108) & 493 & (92) \\
\hline & 506 & (138) & 503 & $(126)$ & 525 & (169) & 528 & (161) \\
\hline & 2086 & (28) & 2003 & (97) & 2334 & $(354)$ & 2128 & $(27)$ \\
\hline & 2020 & $(2646)$ & 2036 & (2143) & 2376 & (3535) & 2163 & $(2625)$ \\
\hline
\end{tabular}


Table S8. The harmonic vibrational frequencies (in $\mathrm{cm}^{-1}$ ) and their IR intensities (in $\mathrm{km} / \mathrm{mol}$ ) for $\mathrm{Rh}_{6}(\mathrm{CO})_{16}$ ( $T_{d}$ tetrabridged isomer).

\begin{tabular}{|c|c|c|c|c|c|c|c|c|}
\hline \multirow[b]{2}{*}{$\mathrm{e}$} & \multicolumn{2}{|c|}{ B3LYP } & \multicolumn{2}{|c|}{ BP86 } & \multicolumn{2}{|c|}{$\mathrm{HF}$} & \multicolumn{2}{|c|}{ MPW1PW91 } \\
\hline & 55 & (0) & 55 & $(0)$ & 54 & (0) & 57 & $(0)$ \\
\hline & 78 & (0) & 76 & (0) & 82 & (0) & 80 & (0) \\
\hline & 120 & (0) & 119 & (0) & 112 & (0) & 130 & (0) \\
\hline & 153 & (0) & 161 & (0) & 133 & (0) & 160 & (0) \\
\hline & 344 & (0) & 355 & (0) & 320 & (0) & 365 & (0) \\
\hline & 410 & (0) & 415 & (0) & 353 & (0) & 428 & (0) \\
\hline & 456 & (0) & 470 & (0) & 423 & (0) & 476 & $(0)$ \\
\hline & 521 & (0) & 515 & (0) & 526 & (0) & 540 & (0) \\
\hline & 2119 & (0) & 2036 & (0) & 2370 & (0) & 2161 & (0) \\
\hline \multirow[t]{7}{*}{$a_{1}$} & 93 & (0) & 90 & (0) & 101 & (0) & 95 & (0) \\
\hline & 183 & (0) & 185 & (0) & 169 & (0) & 200 & (0) \\
\hline & 454 & (0) & 337 & (0) & 327 & (0) & 359 & (0) \\
\hline & 521 & (0) & 475 & (0) & 366 & (0) & 478 & (0) \\
\hline & 542 & (0) & 534 & (0) & 551 & (0) & 561 & (0) \\
\hline & 1904 & (0) & 1816 & (0) & 2157 & (0) & 1943 & (0) \\
\hline & 2172 & (0) & 2083 & (0) & 2435 & (0) & 2215 & (0) \\
\hline \multirow[t]{2}{*}{$\mathrm{a}_{2}$} & 50 & (0) & 49 & (0) & 50 & (0) & 51 & (0) \\
\hline & 413 & (0) & 407 & (0) & 418 & (0) & 428 & (0) \\
\hline \multirow[t]{11}{*}{$\mathrm{t}_{1}$} & 49 & (0) & 48 & (0) & 51 & (0) & 50 & (0) \\
\hline & 54 & (0) & 53 & (0) & 58 & (0) & 56 & (0) \\
\hline & 76 & (0) & 75 & (0) & 82 & (0) & 77 & (0) \\
\hline & 112 & (0) & 111 & (0) & 117 & (0) & 119 & (0) \\
\hline & 146 & (0) & 163 & (0) & 145 & (0) & 159 & (0) \\
\hline & 351 & (0) & 344 & (0) & 311 & (0) & 365 & (0) \\
\hline & 369 & (0) & 376 & (0) & 380 & (0) & 387 & (0) \\
\hline & 404 & (0) & 400 & (0) & 407 & (0) & 419 & (0) \\
\hline & 439 & (0) & 451 & (0) & 433 & (0) & 461 & (0) \\
\hline & 539 & (0) & 537 & (0) & 534 & (0) & 556 & (0) \\
\hline & 2081 & (0) & 1995 & (0) & 2359 & (0) & 2123 & (0) \\
\hline \multirow[t]{16}{*}{$\mathrm{t}_{2}$} & 64 & (0) & 61 & (0) & 67 & (0) & 65 & (0) \\
\hline & 71 & (0) & 70 & (0) & 72 & (0) & 73 & (0) \\
\hline & 88 & (0) & 86 & (0) & 94 & (0) & 90 & (0) \\
\hline & 130 & (0) & 132 & (0) & 125 & (0) & 141 & (0) \\
\hline & 164 & (8) & 169 & (2) & 145 & (20) & 177 & (8) \\
\hline & 175 & (6) & 179 & (6) & 174 & (10) & 191 & (6) \\
\hline & 314 & (13) & 328 & (17) & 234 & (4) & 339 & (14) \\
\hline & 358 & (53) & 364 & (31) & 330 & (18) & 378 & (50) \\
\hline & 401 & (53) & 402 & (73) & 351 & (104) & 419 & (68) \\
\hline & 426 & (42) & 442 & (22) & 363 & (18) & 449 & (44) \\
\hline & 444 & (4) & 461 & (1) & 428 & (58) & 466 & (6) \\
\hline & 501 & (7) & 504 & (12) & 470 & (29) & 520 & (7) \\
\hline & 528 & (147) & 522 & (130) & 535 & $(170)$ & 547 & (162) \\
\hline & 1884 & $(834)$ & 1801 & $(667)$ & 2122 & (1417) & 1921 & $(864)$ \\
\hline & 2100 & (56) & 2014 & (96) & 2364 & (129) & 2142 & (55) \\
\hline & 2134 & $(2865)$ & 2049 & $(2396)$ & 2395 & (3735) & 2177 & $(2862)$ \\
\hline
\end{tabular}

